\title{
Atomic Structure of a Thin Silica Film on a Mo(112) Substrate: A Two-Dimensional Network of $\mathrm{SiO}_{4}$ Tetrahedra
}

\author{
J. Weissenrieder, ${ }^{1}$ S. Kaya, ${ }^{1}$ J.-L. Lu, ${ }^{1,2}$ H.-J. Gao, ${ }^{2}$ S. Shaikhutdinov, ${ }^{1, *}$ H.-J. Freund, ${ }^{1}$ \\ M. Sierka, ${ }^{3}$ T. K. Todorova, ${ }^{3}$ and J. Sauer ${ }^{3}$ \\ ${ }^{1}$ Fritz-Haber-Institut der Max-Planck-Gesellschaft, Faradayweg 4-6, 14195 Berlin, Germany \\ ${ }^{2}$ Institute of Physics, Chinese Academy of Sciences, PO Box 603, Beijing 100080, China \\ ${ }^{3}$ Institut für Chemie, Humboldt Universität zu Berlin, Unter den Linden 6, 10099 Berlin, Germany
} (Received 24 March 2005; published 12 August 2005)

\begin{abstract}
The structure of a thin single crystalline $\mathrm{SiO}_{2}$ film grown on $\mathrm{Mo}(112)$ has been studied by scanning tunneling microscopy, infrared reflection absorption spectroscopy, and x-ray photoelectron spectroscopy. In excellent agreement with the experimental results, density functional theory calculations show that the film consists of a two-dimensional network of corner sharing $\left[\mathrm{SiO}_{4}\right]$ tetrahedra, with one oxygen of each tetrahedron binding to the protruding Mo atoms of the Mo(112) surface.
\end{abstract}

PACS numbers: 68.35. $-\mathrm{p}$, 68.47.Gh, 68.55.- a

Materials with reduced dimensions attract much attention because their properties may differ significantly from the properties of bulk materials. Thin films of dielectric oxides are particularly interesting for a variety of applications that range from insulating layers in integrated circuits to supports for metal nanoparticles in sensors and catalysts. The films can be grown on supporting metal substrates ([1$3]$ and references therein), with a thickness varying from a few angstroms to as much as $100 \AA$, but only in very few cases crystalline films with a known atomic structure are obtained $[4,5]$. In this Letter, based on scanning tunneling microscopy (STM), photoelectron spectroscopy (XPS), infrared reflection absorption spectroscopy (IRAS) and density functional theory (DFT) studies, we report on the atomic structure of a truly two-dimensional crystalline silica on a metal substrate.

The preparation of ordered silica films has only recently been reported [6]. Ricci and Pacchioni have proposed a $\beta$ cristobalite derived structure as the most stable for these films [7]. Based on vibrational spectroscopy data, Goodman and co-workers have recently suggested that the ultra thin silica film consists of a layer of isolated $\mathrm{SiO}_{4}$ clusters arranged in a $c(2 \times 2)$ structure on the $\mathrm{Mo}(112)$ surface, where all oxygen atoms bond to the Mo substrate [8]. Here we show that, when grown on a Mo(112) substrate, silica forms a two-dimensional network of cornersharing $\left[\mathrm{SiO}_{4}\right]$ tetrahedra. One of the oxygen atoms of each tetrahedron binds to the $\operatorname{Mo}(112)$ substrate, while the other three form Si-O-Si bonds with the neighboring tetrahedra. The surface layer is made up of inert Si-O-Si bonds and hence is hydrophobic. Silica surfaces that expose $\mathrm{Si}-\mathrm{O}-\mathrm{Si}$ bonds only, up to now, are exclusively known as internal surfaces of microporous silica modifications $[9,10]$. In contrast, cutting surfaces from bulk silica creates dangling bonds that, depending on external conditions, leads either to surface hydroxylation by water adsorption or to formation of strained $\mathrm{Si}_{-} \mathrm{O}_{2}-\mathrm{Si}$ ("two member") rings by reconstruction [9]. The structure we report here is a new form of silica, which resembles the sheet structure of monolayer silicates [11].

The experiments were performed in an ultrahigh vacuum chamber equipped with STM, IRAS, XPS and low energy electron diffraction (LEED) [6]. The preparation of the films includes Si deposition in an oxygen environment onto an oxygen precovered Mo(112) surface and subsequent annealing in vacuum, resulting in a sharp LEED pattern showing a $c(2 \times 2)-\operatorname{Mo}(112)$ structure $[6,12]$.

Large-scale STM images revealed atomically flat films [Fig. 1(a)], free of holes and silica particles, with terrace steps of $1.2 \AA$ in height, corresponding to the monoatomic steps of the Mo substrate underneath the film.

The high-resolution STM images [see Figs. 1(b) and 1(c)] reveal a honeycomblike structure with a periodicity of $\sim 5.2 \AA$ in the $[\overline{3} 11]$ direction and $\sim 5.5 \AA$ in the $\left[\begin{array}{lll}\overline{1} & \overline{1} & 1\end{array}\right]$ direction, in agreement with $c(2 \times 2)$ structure observed by LEED. In turn, each honeycomb cell is formed by the 6 protrusions separated by $\sim 2.6 \AA$, with the structure depending on the tunneling conditions (cf. Figs. 1(b) and 1(c)]. In addition, an antiphase domain boundary, predicted by Schroeder et al. [6] on the basis of the LEED results, which propagates along the $[\overline{1} 10]$ direction, is seen in Fig. 1(b).

The IRAS spectra for these films show a sharp and strong signal at $1059 \mathrm{~cm}^{-1}\left(\mathrm{FWHM}=12 \mathrm{~cm}^{-1}\right)$ as well as two weak absorption bands at 771 and $675 \mathrm{~cm}^{-1}$ (Fig. 2). The width and position of the main peak was found to be very sensitive to the quality of the film as judged by STM. Using ${ }^{18} \mathrm{O}_{2}$ during the film preparation results in a shift of the vibrational frequencies to $1018 \mathrm{~cm}^{-1}\left(\Delta \nu=41 \mathrm{~cm}^{-1}\right), 764(7)$ and $656(19) \mathrm{cm}^{-1}$, respectively.

The XPS study of these films showed, that the Si $2 p$ region only exhibits a single peak with a binding energy (BE) of $103.2 \mathrm{eV}$, which is typical for a $\mathrm{Si}^{4+}$ oxidation state [13]. The O $1 s$ region, on the other hand, shows two components centered at $531.2 \mathrm{eV}$ and $532.5 \mathrm{eV}$, which 

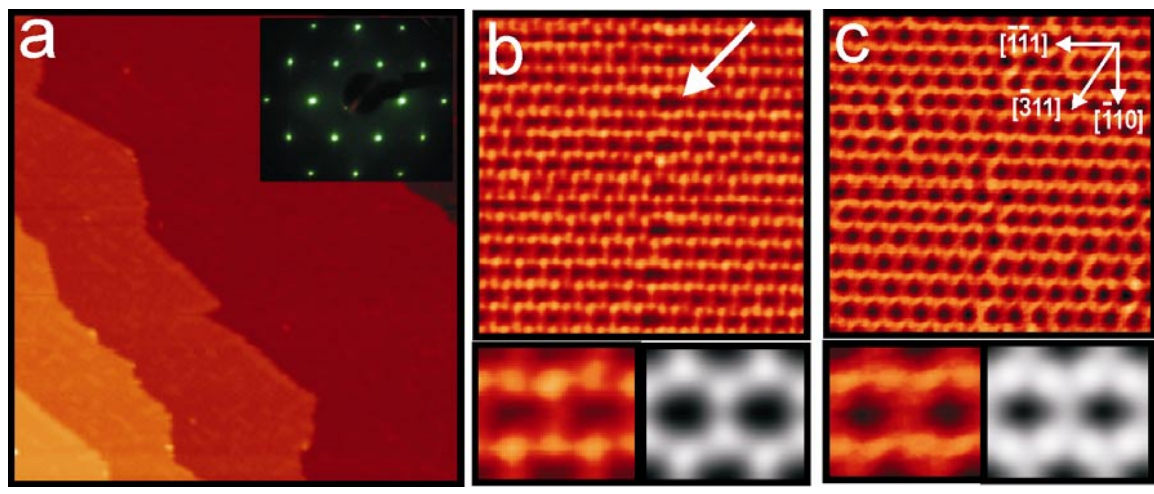

FIG. 1 (color online). STM images of the $\mathrm{SiO}_{2} / \mathrm{Mo}(112)$ film. Size and tunneling parameters: (a) $100 \times 100 \mathrm{~nm}^{2}$, $V_{s}=2 \mathrm{~V}, I=0.2 \mathrm{nA}$, (b) $8 \times 8 \mathrm{~nm}^{2}$, $V_{s}=1.2 \mathrm{~V}, I=0.35 \mathrm{nA}$, (c) $8 \times 8 \mathrm{~nm}^{2}$, $V_{s}=0.65 \mathrm{~V}, I=0.75 \mathrm{nA}$. The arrow indicates the antiphase domain boundary running along the [110] direction. The insets in (b) and (c) show the close up of the atomically resolved STM images (left) and simulated images (right) [the tunneling gap is set $6 \AA$ at $1.2 \mathrm{~V}$ in (b) and $4 \AA$ at $0.65 \mathrm{~V}$ in (c)].

suggest the presence of two oxygen species. Both BEs are significantly higher than those observed for the $\mathrm{MoO}_{x}$ oxide films formed on the Mo(112) surfaces $(\sim 530 \mathrm{eV}$ [14]), and therefore cannot be assigned to the oxidation of the Mo substrate underneath the film.

The combined experimental results suggest that: (i) A silica network rather than isolated $\left[\mathrm{SiO}_{4}\right]$ clusters, as proposed by Goodman's group [8], is formed. (ii) There are two oxygen species residing in different chemical environments, one of which can reasonably be assigned to oxygen binding to the substrate, and the other one to bridging oxygen atoms surrounded only by the $\mathrm{Si}^{4+}$ cations. (iii) There is a vibration of a bond with a dipole moment perpendicular to the surface. The asymmetric stretching vibration of $\mathrm{Si}-\mathrm{O}-\mathrm{Mo}$ species is a good candidate for such a mode, as suggested by Chen et al. [8]. Concomitantly, the weaker bands at 771 and $675 \mathrm{~cm}^{-1}$ correspond to the vibrational modes with a small net dipole moment normal to the surface. (iv) The surface is coordinatively saturated and oxygen terminated, based on the inertness of the film towards $\mathrm{CO}, \mathrm{O}_{2}$, and water (not shown here, see also [15]).

Inspired by the structures of layered silicates [11] we have constructed more than 15 models consisting of single and double layers of $\left[\mathrm{SiO}_{4}\right]$ tetrahedra, which do not have dangling $\mathrm{Si}-\mathrm{O}$ bonds or edge-shared $\mathrm{SiO}_{4}$ tetrahedra strained (two membered silica rings) at the film surface. We employed DFT [16] to examine their structural stability for a fully relaxed geometry. It has turned out that the most stable are single-layer structures with a surface unit cell composition of $\mathrm{Si}_{4} \mathrm{O}_{10}$, which form a network of cornersharing $\left[\mathrm{SiO}_{4}\right]$ tetrahedra (Fig. 3). One corner of each tetrahedron is pointing to the underlying Mo substrate thus forming a Si-O-Mo bond. (It should be mentioned that these structures show some similarities with the proposed, but rejected model 4 of Ref. [8]). The three models presented in Fig. 3 differ by the adsorption site of the interface $\mathrm{O}$ atom on $\mathrm{Mo}(112)$, i.e., bridge (model $A$ ), atop $(B)$, or pseudo three-fold hollow $(C)$ sites with respect to the protruding Mo atoms running along the [1 111$]$ direction. In line with LEED data, all models show a $c(2 \times$ 2) structure with respect to $\mathrm{Mo}(112)$.
The stability of the $\mathrm{SiO}_{2} / \mathrm{Mo}(112)$ films was compared using the energy, $\Delta E_{\text {form }}$, of its hypothetical formation from the clean $\operatorname{Mo}(112)$ surface, bulk $\alpha$-quartz, and oxygen:

$$
\begin{aligned}
\mathrm{Mo}(112)+m\left(\mathrm{SiO}_{2}\right)_{\alpha-\text { quartz }} & \\
& +n \frac{1}{2} \mathrm{O}_{2} \rightarrow\left(\mathrm{SiO}_{2}\right)_{m} n \mathrm{O} / \mathrm{Mo}(112)
\end{aligned}
$$

where $m$ and $n$ are the number of $\mathrm{SiO}_{2}$ units and oxygen

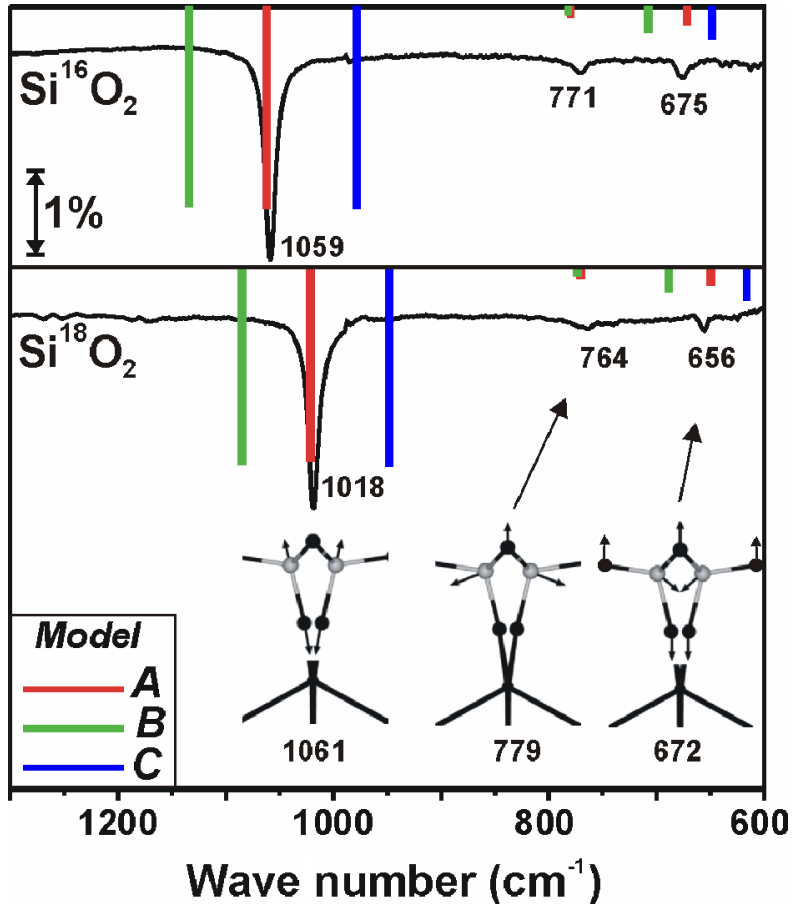

FIG. 2 (color online). IRAS-spectra of silica films prepared with ${ }^{18} \mathrm{O}$ and ${ }^{16} \mathrm{O}$. Calculated frequencies of the three IR active vibrations are represented by bars for three different models shown in Fig. 3, with the height proportional to the intensity

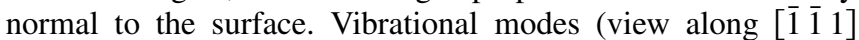
direction) of the most stable structure $A$ are schematically shown. $\mathrm{O}$ and $\mathrm{Si}$ are represented by black and gray spheres, respectively. For clarity the $\operatorname{Mo}(112)$ surface is drawn as sticks. 


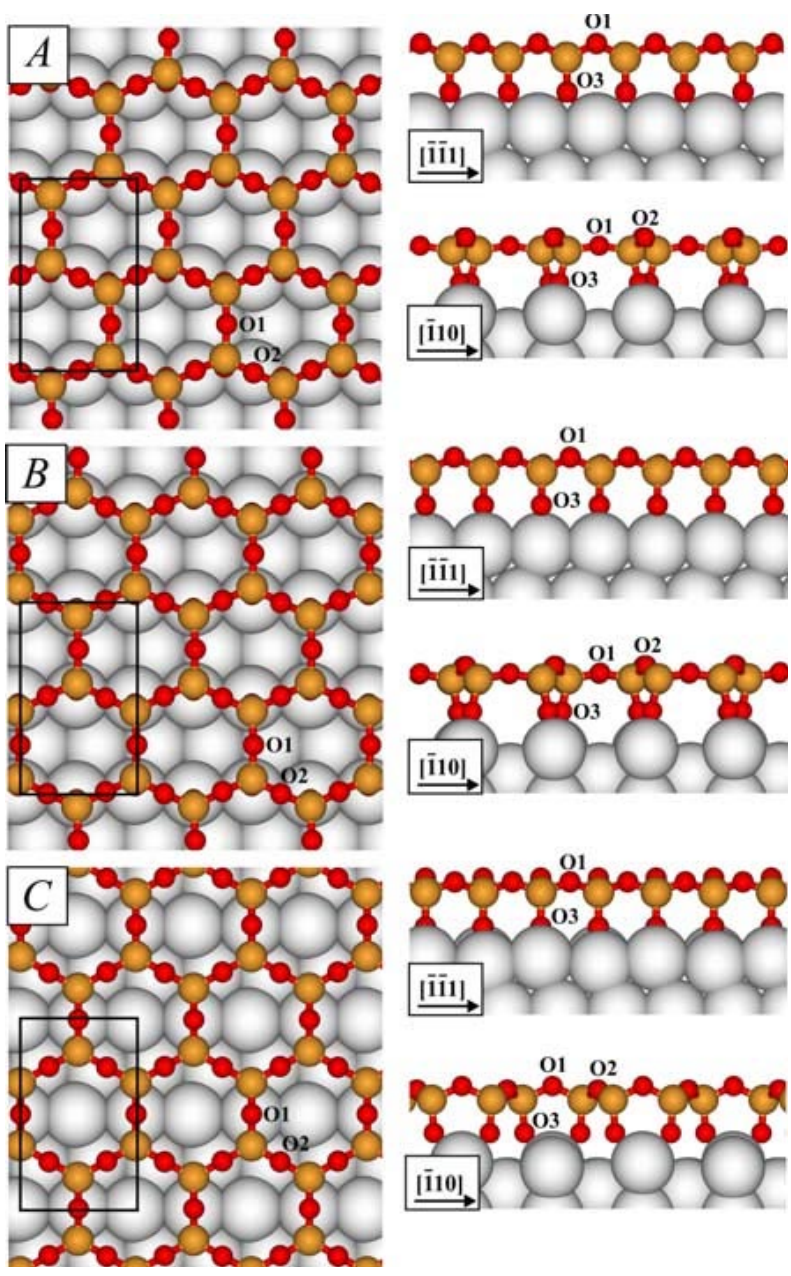

FIG. 3 (color online). Three models of the single-layer $\mathrm{SiO}_{2} / \mathrm{Mo}(112)$ film. The $\mathrm{Si}_{4} \mathrm{O}_{10}$ surface unit cell is indicated.

excess in a surface unit cell, respectively. With this definition the model $A$ is about $2 \mathrm{eV}\left(\Delta E_{\text {form }}=-10.8 \mathrm{eV}\right)$ more stable than $B(-8.8 \mathrm{eV})$ and $C(-8.7 \mathrm{eV})$. The $\beta$-cristobalite (three-layer) film structure proposed by Ricci and Pacchioni [7] is $1.7 \mathrm{eV}$ less stable $(m=10$, $n=3, \Delta E_{\text {form }}=-9.1 \mathrm{eV}$ ) than model $A$, while the monolayer of isolated $c(2 \times 2)$ - $\left[\mathrm{SiO}_{4}\right]$ clusters proposed by Chen et al. [8] is slightly more stable $(m=2, n=4$, $\Delta E_{\text {form }}=-11.5 \mathrm{eV}$ ). However, as the compositions of these structures are different from that of our films, the relative stability must be compared at a given $\mathrm{O}_{2}$ partial pressure. This is included in the free energy of reaction (1) per surface area $S[23,24]$,

$$
\Delta \gamma(T, p)=\frac{1}{S}\left[\Delta E_{\text {form }}-n \frac{1}{2} \Delta \mu_{\mathrm{O}}(T, p)\right]
$$

with $\Delta \mu_{\mathrm{O}}(T, p)=\mu_{\mathrm{O}}-1 / 2 E_{\mathrm{O}_{2}}, E_{\mathrm{O}_{2}}$ is the energy of an $\mathrm{O}_{2}$ molecule and $\mu_{\mathrm{O}}$ is the oxygen chemical potential that relates to pressure [24]. Our calculations show that the structure $A$ is thermodynamically the most favorable at all experimentally relevant oxygen pressures.
In addition, we have performed calculations of the vibrational spectra for the models $A, B$, and $C$ (for details see $[25,26])$. The results revealed only three IR active modes above $400 \mathrm{~cm}^{-1}$. The most intense mode is observed for the asymmetric Si-O-Mo stretching at $1061 \mathrm{~cm}^{-1}$ originating from the $\mathrm{Si}-\mathrm{O}$ bond pointing downwards to the Mo substrate. The second mode at $779 \mathrm{~cm}^{-1}$ is the $\mathrm{Si}-\mathrm{O}-\mathrm{Si}$ symmetric stretching coupled with $\mathrm{Si}-\mathrm{O}-\mathrm{Si}$ bending and the third mode at $672 \mathrm{~cm}^{-1}$ is a coupling of $\mathrm{Si}-\mathrm{O}-\mathrm{Si}$ bending modes. Figure 2 demonstrates that the positions and relative intensities of the bands for the structure $A$ are in excellent agreement with the experimental results, while the other two models disagree by $\sim 75 \mathrm{~cm}^{-1}$ for the main peak. The calculations correctly predict also the magnitude of isotopic shifts observed for the films grown with ${ }^{18} \mathrm{O}_{2}$. On the contrary, the vibrational spectrum calculated for the film of isolated $\left[\mathrm{SiO}_{4}\right]$ clusters [8] shows two almost equally intense peaks at 706 and $532 \mathrm{~cm}^{-1}$, which clearly disagree with the experimental spectra.

Furthermore, for these three models we have calculated the difference in BEs of $\mathrm{O} 1 s$ core levels between different oxygen atoms present in the film [27]. For the $\mathrm{O} 1$ and $\mathrm{O} 2$ atoms in Si-O-Si bridging positions [see Figs. 3(a)-3(c), the BEs are nearly equal $( \pm 0.1 \mathrm{eV})$. The BE difference between $\mathrm{O} 1(\mathrm{O} 2)$ and $\mathrm{O} 3$ atoms is much larger and found to be $1.3,1.9$, and $1.1 \mathrm{eV}$ for the models $A, B$, and $C$, respectively. The experimentally observed value $(1.3 \mathrm{eV})$ again favors model $A$. Therefore, our calculations not only prove the single-layer network as the most stable structure of the silica film but also determine the adsorption sites for interfacial oxygen bonded to the $\operatorname{Mo}(112)$ surface.

Finally, based on a calculated density of states, we have simulated STM images of the most stable structure at tunneling conditions used in the experiments based on the Tersoff-Hamann approach [28]. Interestingly, the protrusions imaged by STM may be attributed to the Si atoms at a large tip-surface distance or to the bridging oxygen atoms at a smaller distance (see insets in Figs. 1(b) and 1(c)]. In both cases, the simulated images match well the experimental ones, thus providing further strong evidence for the structure model $A$ determined by IRAS and XPS.

In summary, based on the excellent agreement between experimental and theoretical results, we have determined the atomic structure of the well-ordered silica film on Mo(112), which consists of a two-dimensional network of corner-sharing $\left[\mathrm{SiO}_{4}\right]$ tetrahedra. The structure explains the essential inertness of the silica films, in particular, towards water [15]. The knowledge of the atomic structure of the silica film puts us into the position to study nucleation and growth of metals on flat well characterized silica surfaces at the atomic level.

We thank G. Kresse for providing software for simulations of the STM images and M. V. Ganduglia-Pirovano for helpful discussions. The calculations were carried out at the IBM p690 system of the Norddeutscher Verbund für 
Hoch- und Höchstleistungsrechnen (HLRN). Financial support by Deutsche Forschungsgemeinschaft (SFB546) is acknowledged, and J.W. thanks the Alexander von Humboldt Foundation for support.

Note added. - After the manuscript was ready for submission we learned that G. Pacchioni and co-workers independently found that single-layer $\mathrm{SiO}_{4}$ networks are the most stable structures of the $\mathrm{SiO}_{2} / \mathrm{Mo}(112)$ film [29].

*Corresponding author.

Electronic address: shaikhutdinov@fhi-berlin.mpg.de

[1] D. W. Goodman, Surf. Rev. Lett. 2, 9 (1995).

[2] R. Franchy, Surf. Sci. Rep. 38, 199 (2000).

[3] H.-J. Freund, Angew. Chem., Int. Ed. Engl. 36, 452 (1997).

[4] G. Kresse, M. Schmid, E. Napetschnig, M. Shishkin, L. Köhler, and P. Varga, Science (to be published).

[5] F. Rohr, M. Bäumer, H.-J. Freund, J. A. Meijas, V. Staemmler, S. Müller, L. Hammer, and K. Heinz, Surf. Sci. Lett. 372, L291 (1997); 389, 391 (1997).

[6] T. Schroeder, M. Adelt, B. Richter, M. Naschitzki, J. B. Giorgi, M. Bäumer, and H.-J. Freund, Surf. Rev. Lett. 7, 7 (2000).

[7] D. Ricci and G. Pacchioni, Phys. Rev. B 69, 161307 (2004).

[8] M. S. Chen, A. K. Santra, and D.W. Goodman, Phys. Rev. B 69, 155404 (2004).

[9] E. Flanigan, J. M. Bennett, R. W. Grose, J. P. Cohen, R. L. Patton, R. M. Kirchner, and J. V. Smith, Nature (London) 271, 512 (1978).

[10] M.E. Davis, Nature (London) 417, 813 (2002).

[11] F. Liebau, Structural Chemistry of Silicates (SpringerVerlag, Berlin, 1985).

[12] The Mo(112) single crystal was cleaned by annealing in oxygen and subsequent flashing to above $2300 \mathrm{~K}$. The impurity level was below the detection limit of XPS. The clean $\mathrm{Mo}(112)$ surface was exposed to $5 \times 10^{-8} \mathrm{mbar}_{2}$ at $850 \mathrm{~K}$ for $5 \mathrm{~min}$ and then approximately 2 monolayers of $\mathrm{Si}$ was deposited at the same temperature and pressure for $15 \mathrm{~min}$. The film was subsequently annealed at $1200 \mathrm{~K}$ in vacuum until a sharp $c(2 \times 2)-\mathrm{Mo}(112)$ LEED pattern was observed.

[13] J.F. Moulder, W.F. Stickle, P.E. Sobol, and K.D. Bomben, Handbook of X-Ray Photoelectron Spectroscopy (Perkin-Elmer Corporation USA, Minnesota, 1992).

[14] T. Schroeder, J. B. Giorgi, A. Hammoudeh, N. Magg, M. Bäumer, and H.-J. Freund, Phys. Rev. B 65, 115411 (2002).
[15] S. Wendt, M. Frerichs, T. Wei, M.S. Chen, V. Kempter, and D. W. Goodman, Surf. Sci. 565, 107 (2004).

[16] The calculations were performed using the Vienna $A b$ initio Simulation Package (VASP) $[17,18]$ and the projector augmented wave method $[19,20]$ together with the PW91 exchange-correlation functional [21]. The Mo(112) slabs were modeled using orthorhombic $(2 \times$ 2) supercell, $a_{0}=5.464 \AA$ and $b_{0}=8.923 \AA$, containing seven Mo layers, three of them were fixed to their bulk positions and four were relaxed. We used $400 \mathrm{eV}$ cutoff for the plane waves basis set. The integrations in the Brillouin zone were performed using a $(8 \times 4 \times 1)$ Monkhorst-Pack grid [22].

[17] G. Kresse and J. Furthmüller, Comput. Mater. Sci. 6, 15 (1996).

[18] G. Kresse and J. Furthmüller, Phys. Rev. B 54, 11169 (1996).

[19] P. E. Blöchl, Phys. Rev. B 50, 17953 (1994).

[20] G. Kresse and D. Joubert, Phys. Rev. B 59, 1758 (1999).

[21] J. P. Perdew et al., Phys. Rev. B 46, 6671 (1992).

[22] H. J. Monkhorst and J. D. Pack, Phys. Rev. B 13, 5188 (1976).

[23] Vibrational contributions of the solid components are neglected; i.e., their free energies are replaced by the energies.

[24] K. Reuter and M. Scheffler, Phys. Rev. B 65, 035406 (2002).

[25] We used the harmonic approximation within the central finite difference method with $0.02 \AA$ displacements. The intensities are obtained from the derivatives of the dipole moment component perpendicular to the surface.

[26] See EPAPS Document No. E-PRLTAO-95-063534 for details of the frequency calculations and comparison of the calculated and experimental values for alpha quartz as the reference substance. This document can be reached via a direct link in the online article's HTML reference section or via the EPAPS homepage (http://www.aip.org/ pubservs/epaps.html).

[27] Calculations of the total core-level shifts are performed using modified projector augmented wave potential. The core hole is created by removing half of an electron from the oxygen $1 s$ state. Final states are approximated using the Slater transition state concept: J. C. Slater, in Quantum Theory of Molecules and Solids (McGraw-Hill, New York, 1974), Vol. 4.

[28] J. Tersoff and D. R. Hamann, Phys. Rev. B 31, 805 (1985).

[29] L. Giordano, D. Ricci, G. Pacchioni, and P. Ugliengo, Surf. Sci. (to be published). 